\title{
Effects of switching ticagrelor to clopidogrel on cardiovascular outcomes in patients with acute coronary syndrome: Erratum
}

In the article, "Effects of switching ticagrelor to clopidogrel on cardiovascular outcomes in patients with acute coronary syndrome", ${ }^{[1]}$ which appeared in Volume 97, Issue 48 of Medicine, Dr. Jianping Liu's name appeared incorrectly as Lin Liu.

\section{Reference}

[1] Liu L, Liao H, Zhong S. Effects of switching ticagrelor to clopidogrel on cardiovascular outcomes in patients with acute coronary syndrome. Medicine. 97;48: e13381. 